\title{
Rapid Sampling of Molecules via Skin for Diagnostic and Forensic Applications
}

\author{
Sumit Paliwal • Makoto Ogura $\cdot$ Samir Mitragotri
}

Received: 20 October 2009 / Accepted: 9 February 2010 / Published online: 18 March 2010

(C) The Author(s) 2010. This article is published with open access at Springerlink.com

\begin{abstract}
Purpose Skin provides an excellent portal for diagnostic monitoring of a variety of entities; however, there is a dearth of reliable methods for patient-friendly sampling of skin constituents. This study describes the use of low-frequency ultrasound as a one-step methodology for rapid sampling of molecules from the skin.
\end{abstract}

Methods Sampling was performed using a brief exposure of $20 \mathrm{kHz}$ ultrasound to skin in the presence of a sampling fluid. In vitro sampling from porcine skin was performed to assess the effectiveness of the method and its ability to sample drugs and endogenous epidermal biomolecules from the skin. Dermal presence of an antifungal drug-fluconazole and an abused substance, cocaine-was assessed in rats.

Results Ultrasonic sampling captured the native profile of various naturally occurring moisturizing factors in skin. A high sampling efficiency $(79 \pm 13 \%)$ of topically delivered drug was achieved. Ultrasound consistently sampled greater amounts of drug from the skin compared to tape stripping. Ultrasonic sampling also detected sustained presence of cocaine in rat skin for up to 7 days as compared to its rapid disappearance from the urine.

Conclusions Ultrasonic sampling provides significant advantages including enhanced sampling from deeper layers of skin and high temporal sampling sensitivity.

KEY WORDS drugs · forensic · sampling · tape stripping · ultrasound

Sumit Paliwal and Makoto Ogura contributed equally.

S. Paliwal $\cdot$ M. Ogura $\cdot$ S. Mitragotri $(\bowtie)$

Department of Chemical Engineering, University of California,

Santa Barbara, California 93106, USA

e-mail: samir@engineering.ucsb.edu

\section{INTRODUCTION}

Skin, the outermost organ of the human body, acts as a reservoir for chemicals that come in its contact either because of topical drug application or because of unintended exposure to entities such as pollutants or industrial chemicals (1). Several systemically administrated chemicals, including therapeutic and abused drugs, also accumulate in the skin over a period of time (2-5). Accordingly, a quantitative assessment of skin's chemical composition has potential applications in varied fields, including therapeutic drug monitoring and exposure assessment. However, the lack of reliable methods for standardized and patientfriendly sampling of skin's constituents has significantly hampered translation of skin-based diagnostic methods into the clinic (6).

Over the years, a handful of methods have been reported for direct sampling of molecular components of the skin. Earliest methods include removal of skin strata by shave/punch biopsies and analyses of molecular constituents in the liquefied biopsy samples (7). Methods have also been developed to harvest skin's interstitial fluid through suction-blisters or microdialysis (8). However, applications of these techniques in the clinic have been limited by their invasiveness and practical challenges. As opposed to these invasive methods of varying degree, the tape-stripping technique, which requires an adhesive to bind and collect the contents of the skin surface, has been used for sampling skin, specifically its outermost layer-stratum corneum (SC) (9). Traditionally, the tape-stripping method has been reported to be limited by lack of a standardized protocol $(10,11)$ and high site-to-site sampling heterogeneity (12); however, several recent studies have advanced this technique to more accurately determine the pharmacokinetic profile of drugs in skin and control the sampling variability (13-17). 
Among other techniques, reverse iontophoresis, which applies low intensities of current through skin, has reported excellent capabilities to sample glucose, lithium, phenytoin, valproate, lactate and urea from the skin (18). In another technique, low-frequency ultrasound has also been used to sample interstitial fluid through a two-step process: first, by permeabilizing the skin by ultrasound and subsequently, by applying vacuum to collect interstitial fluid (19-21).

Herein, we describe the use of low-frequency ultrasound as a rapid and one-step sampling methodology for determination of chemicals. We specifically describe quantitative sampling of skin's natural moisturizing factors (a family of molecules critical for skin's hydration, mechanical strength and metabolic activities) as well as exogenous therapeutic drug molecules and drugs-of-abuse in the skin. Detailed in vitro and in vivo experimental results characterizing this methodology's sampling efficiency as compared with tape-stripping technique are also presented.

\section{METHODS}

\section{Skin Procurement and Animals Models}

In vitro sampling experiments were performed on porcine skin. Full-thickness porcine skin was harvested from the lateral abdominal region of Yorkshire pigs, adipose tissue was stripped and skin was sectioned into $10 \mathrm{~cm} \times 25 \mathrm{~cm}$ pieces by Lampire Biological Laboratories Inc., PA. Skin pieces were shipped over dry ice and stored at $-70^{\circ} \mathrm{C}$ upon receipt until the experiment. Skin pieces with no visible imperfections such as scratches and abrasions were thawed at room temperature and used for experiments. Skin was further cut into small pieces $(2.5 \mathrm{~cm} \times 2.5 \mathrm{~cm})$.

In vivo experiments were performed using Female Sprague Dawley rats (8-10 weeks, Charles River Laboratory, Wilmington, MA). All animal handling and maintenance protocols were approved by the Institutional Animal Care and Use Committee, University of California, Santa Barbara, CA.

\section{One-Step Ultrasonic Sampling Procedure}

Earlier studies with ultrasound focused on sampling interstitial fluid through a two-step process: permeabilizing the skin by ultrasound and subsequently applying vacuum to collect interstitial fluid. In this study, we describe a new direct sampling approach where skin constituents are collected into the ultrasound coupling medium during the sonication procedure itself. In vitro experiments were carried out by a brief application of ultrasound $\left(20 \mathrm{kHz}, 2.4 \mathrm{~W} / \mathrm{cm}^{2}\right.$, $50 \%$ duty cycle, Sonics \& Materials, Newtown, CT) to skin mounted on a Franz diffusion cell assembly (skin exposure area of $1.77 \mathrm{~cm}^{2}$; Permegear, Hellertown, PA). The receiver chamber of the diffusion cells was filled with phosphate-buffered saline (PBS) (P4417, Sigma-Aldrich, St. Louis, MO), and the donor chamber was filled with $1 \mathrm{ml}$ of sampling medium $(1 \% \mathrm{w} / \mathrm{v}$ sodium lauryl sulfate (SLS) in sterile PBS), which also functioned as the coupling fluid between the ultrasound transducer and skin. For in vivo experiments, animals were anesthetized with 1.25-4\% isofluorane in oxygen and, shaved on the abdomen or back with a clipper, and a custom-made flanged chamber (skin exposure area of $1.33 \mathrm{~cm}^{2}$ ) was glued to the shaven area with a cyanoacrylate-based adhesive. The chamber was filled with $1.5 \mathrm{ml}$ of sampling medium. Transepidermal current was measured by applying $143 \mathrm{mV}$ AC signal at $10 \mathrm{~Hz}$ (33120A, Agilent, Santa Clara, CA) across the sonicated skin to determine the extent of skin permeabilization. For in vitro experiments, electrodes (4-mm $\mathrm{Ag} / \mathrm{AgCl}$ disk electrode, Invivo Metrics, Healdsburg, CA) were inserted in the donor and receiver chambers of the Franz diffusion cell assembly. Only those skin pieces having an initial resistivity of $30 \mathrm{k} \Omega-\mathrm{cm}^{2}$ or more were used in the experiments. For in vivo experiments, an electrode was introduced in the sampling medium, and a subcutaneous reference was used as the counter electrode. Should this method be applied to humans, a subcutaneous reference will not be necessary and can be replaced by a topical patch electrode. Initial rat skin resistivity was about $100 \mathrm{k} \Omega-\mathrm{cm}^{2}$. A 20-fold increase in transepidermal current (equivalent to about 20-fold decrease in skin resistivity) was chosen in this study as a threshold to stop ultrasound application and ensure safety of ultrasound exposure. A 20-fold increase in skin conductivity typically required about 5 min of ultrasound exposure at 50\% duty cycle. All experiments were carried out at room temperature. Ultrasonic exposure is often associated with thermal effects. The magnitude of thermal effects depends on several parameters, including ultrasound intensity, duty cycle, exposure time, and volume of the sampling medium. While a moderate temperature increase is acceptable, these effects were minimized so as to avoid confounding contributions by thermal and mechanical effects. As such, ultrasound under the conditions used in this study has been previously used in humans without any adverse thermal effects (22). During the sampling procedure, care was taken to minimize the thermal effects of ultrasound on skin. The temperature of the sampling medium was monitored after each minute of ultrasound exposure using a thermocouple. If temperature of the medium increased over $5^{\circ} \mathrm{C}$, the sampling medium was aspirated in a centrifuge tube and briefly chilled over an ice bath for $1 \mathrm{~min}$. Most of the experiments did not require temperature modulation; however, when necessary, a single cooling procedure was sufficient. After the completion of the sonication procedure, sampling medium was immediately aspirated using a Pasteur pipette and stored at $-20^{\circ} \mathrm{C}$ for later analysis of the collected analytes. 
In experiments requiring a comparative analysis of molecules retrieved by ultrasonic sampling with the actual composition of molecules present in the skin, epidermal skin homogenate samples were prepared from heat-stripped epidermal (stratum corneum and viable epidermis) porcine skin. Skin was heat-stripped using a microwave oven for $5 \mathrm{~s}$, and full epidermis was carefully stripped off from the skin using a pair of tweezers.

\section{Analysis of Natural Moisturizing Factors}

Ultrasonic samples $(n=5)$ were analyzed to quantify the presence of natural moisturizing factors. Epidermal homogenates were used as positive controls $(n=5)$. Passive sampling was performed by incubation of the sampling medium with skin for $5 \mathrm{~min}(n=5)$. The samples were deproteinized by adding $2.5 \% \mathrm{v} / \mathrm{v}$ (final concentration) trichloroacetic acid followed by centrifugation to retrieve the acid-soluble supernatants (as previously described in Ref. (23)). Acid in the samples was then neutralized to $\mathrm{pH} 7.2$ by adding $1 \mathrm{~N} \mathrm{NaOH}$. Amino acid analysis of the samples was done by ion exchange HPLC with ninhydrin-based detection system (6300 Auto Analyzer, Beckman Coulter, Fullerton, CA), and absorbance was processed by HP ChemStation (A.06.03(509), Hewlett-Packard, Wilmington, DE) in external standard mode using a standard amino acid mixture (AAS18, Sigma-Aldrich, St. Louis, MO). Specifically, aspartic acid (asp), threonine (thr), serine (ser), glutamic acid (glu), proline (pro), glycine (gly), alanine (ala), cysteine (cys), valine (val), methionine (met), isoleucine (ile), leucine (leu), phenylalanine (phe), tyrosine (tyr), histidine (his), lysine (lys), and arginine (arg) were analyzed. UV-Visual spectroscopy-based commercial kits were bought and used according to the manufacturer's instruction, for the quantification of lactic acid (K-DLATE, Megazyme, Wicklow, Ireland; $n=4$ ) and urea (DIUR-500, BioAssay Systems, Hayward, CA, $n=4$ ) in the samples.

\section{Delivery and Detection of Drugs in Skin}

In vitro experiments on porcine skin were designed using Franz diffusion cells to assess delivery of radiolabeled docosanol $(\log$ P: 10.5, Molecular weight: 326.57 Da; ART 0449, American Radiolabeled Chemicals, St. Louis, MO) in the skin. Docosanol was topically delivered by applying the drug solution in PBS for $4 \mathrm{~h}$ (donor drug concentration: $10 \mu \mathrm{Ci} / \mathrm{ml})$. At the end of the experiment, to ensure removal of residual drug on skin, the formulation was aspirated from the donor chamber, and the chamber was briefly washed by repeatedly dispensing and aspirating $1 \mathrm{ml}$ of saline with a Pasteur pipette. This procedure was repeated three times with fresh saline. Skin was tape-stripped $\left(\mathrm{S} \operatorname{cotch} \circledast{ }^{\circledR}\right.$ Magic ${ }^{\mathrm{TM}}$ tape, 3 M, St. Paul, MN) two times to further remove residual drug on the skin. These tape strips were discarded and not included in the drug analysis. Skin was sampled ultrasonically $(n=3)$ as described above or with tape stripping $(n=3)$ in separate experiments. Decrease in skin's electrical impedance was used as an indirect measure for the amount of stratum corneum sampled by tape-stripping procedure. Saturation in the decrease of skin's electrical resistivity after each tape strip-defined as less than 10\% decrease in electrical resistivity between two sequential tape strips and representative of nearly complete removal of stratum corneum - was chosen as the endpoint of the tape-stripping procedure. For the batch of abdominal porcine skin used in the in vitro experiments, it took about 25 tape strips to reach saturation. Tape strips were analyzed in five groups consisting of five tapes each. Each group of tapes was incubated overnight with $5 \mathrm{ml}$ of tissue solvent (Solvable, Catalog No. 6NE9100, PerkinElmer, Waltham, MA) at room temperature to extract drug from the tapes. Extraction efficiency of this procedure was determined to be $93.1 \pm 7.3 \%$. Passive sampling $(n=3)$ was also performed by incubating the sampling medium for 5 min with skin.

In two additional sets of experiments, docosanol was subdermally delivered for either $8 \mathrm{~h}$ or $24 \mathrm{~h}$ by adding drug solution in the receiver compartment of the diffusion cell (receiver drug concentration: $1 \mu \mathrm{Ci} / \mathrm{ml}$ ). After the drug was delivered, ultrasonic sampling $(n=3)$ and passive sampling $(n=3)$ were performed from the donor chamber. Tapestripping procedure (total 25 tape strips, $n=3$ ) was also performed. Since drug was not introduced in the donor, initial tapes were not discarded and were included in the analysis.

For in vivo drug bioavailability assessment, Sprague Dawley rats were placed in a wire mesh cage which allowed animal excreta to be collected in a separate plate placed underneath the cage. Such an arrangement minimized contamination of skin with urine. Rats were intravenously administered with $10 \mathrm{mg} / \mathrm{kg}$ fluconazole along with $250 \mu \mathrm{Ci} / \mathrm{kg}$ of radiolabeled fluconazole $(\log P$ : 0.4, Molecular weight: 306.27 Da; MT 1752, Moravek Radiochemicals, Brea, CA) or $5 \mathrm{mg} / \mathrm{kg}$ cocaine along with $175 \mu \mathrm{Ci} / \mathrm{kg}$ of radiolabeled cocaine (log P: 2.3, Molecular weight: 303.35 Da; ART 0651, American Radiolabeled Chemicals, St. Louis, MO) by tail vein injections in two separate experiments. Since dosage directly affects the rate of drug metabolism and its distribution in vivo, nonradiolabeled drug was added to the formulation to administer physiologically relevant doses of fluconazole and cocaine in animals. The drugs were sampled from the back of the animals at various times after the injection, with each sampling performed at a different skin site. Nine experiments were performed corresponding to three animals and three distinct skin sites per animal. Urine samples and blood samples (drawn through the jugular vein) were also simultaneously collected to compare with ultrasonic skin sampling technique. Topical skin swabs were carried out as a passive 
control using a cotton ball soaked in $500 \mu \mathrm{l}$ of $1: 1(\mathrm{v} / \mathrm{v})$ mixture of water and ethanol, and cynoacrylate tape-stripping was done to collect drug from the full epidermis.

\section{Statistical Analysis}

Student's $t$-test was applied to test if there were any significant differences between the amount of molecules sampled by ultrasound and different types of samples, such as skin homogenate, blood, urine and samples retrieved by tape stripping.

\section{RESULTS}

\section{Sampling of Endogenous Small Molecules of Skin}

The ability of ultrasound to sample endogenous epidermal biomolecules from skin was assessed in vitro. For this purpose, we focused on assessing the presence of natural moisturizing factors $(\mathrm{NMFs})$ in skin. NMFs are comprised of many molecules, of which free amino acids, lactate and urea that collectively constitute over $60 \% \mathrm{w} / \mathrm{w}$ of the total NMFs present in the skin (24), were detected in this study. A quantitative analysis was performed to compare ultrasonically sampled amino acids with their native composition profile in the epidermal skin. Table I shows the concentration (nmole per $\mathrm{cm}^{2}$ skin) of 16 most abundant free amino acids in ultrasound sample, passive sample and epidermal skin homogenate. With the exception of glutamic acid and arginine $(p<0.05$, Student's $t$-test, $n=5)$, a good overall correlation between the relative amount of amino acids (\% mole/mole) in the ultrasonic sample and epidermal homogenate was observed. Ultrasound sampled $15.7 \pm 4.7 \%$ of amino acids present in the epidermal skin. Only 7 amino acids were sampled passively and at much lower concentrations as compared with ultrasound samples. Other NMFs, including lactic acid and urea were also detected in the ultrasound sample of porcine skin at concentrations of $41.82 \pm$ $26.65 \mathrm{nmole} / \mathrm{cm}^{2}$ and $13.92 \pm 5.07 \mu$ mole $/ \mathrm{cm}^{2}$, respectively.

\section{Assessment of Drug Delivered to Skin After Topical and Systemic Administration}

The ability of the ultrasonic sampling method to determine the amount of drug accumulated in the skin was assessed. A model drug, docosanol, was first topically delivered for $4 \mathrm{~h}$ into porcine skin mounted on a Franz diffusion cell and was later sampled using ultrasound. Ultrasound recovered $79 \pm 13 \%$ of the drug accumulated in the epidermis, as determined by the drug concentration in the epidermis homogenate $(n=5)$. Effectiveness of ultrasonic sampling was compared with that of the tape-stripping technique. A significant amount of docosanol was collected in the first five strips; however, the cumulative amount of docosanol sampled by 25 tape strips was only $40 \%$ of that sampled by ultrasound $(p<0.001$, Student's $t$-test, $n=3$; Fig. 1a). Higher recovery of docosanol by ultrasound suggests

Table I Amount of Amino Acids Present in Epidermal Homogenate and in Samples Acquired by Passive Sampling and Ultrasonic Sampling

\begin{tabular}{|c|c|c|c|c|c|}
\hline \multirow[t]{2}{*}{ AA } & \multicolumn{2}{|c|}{ AA sampled by ultrasound } & \multicolumn{2}{|c|}{ AA in epidermal homogenate } & \multirow[t]{2}{*}{ AA sampled passively $\left(\mathrm{nmol} / \mathrm{cm}^{2}\right)$} \\
\hline & $\left(\mathrm{nmol} / \mathrm{cm}^{2}\right)$ & $\%$ (mole/mole) & $\left(\mathrm{nmol} / \mathrm{cm}^{2}\right)$ & $\%$ (mole/mole) & \\
\hline asp & $3.7 \pm 1.0$ & $3.5 \pm 1.2$ & $32.0 \pm 19.0$ & $4.2 \pm 1.5$ & $2.4 \pm 0.6$ \\
\hline thr & $5.5 \pm 1.9$ & $5.1 \pm 1.5$ & $49.8 \pm 15.0$ & $7.1 \pm 2.7$ & $2.1 \pm 0.6$ \\
\hline ser & $19.0 \pm 9.9$ & $17.8 \pm 8.8$ & $161.7 \pm 18.3$ & $23.2 \pm 4.5$ & $7.3 \pm 1.3$ \\
\hline glu & $4.5 \pm 1.1$ & $4.2 \pm 0.7^{a}$ & $45.5 \pm 14.3$ & $6.3 \pm 1.5$ & $2.5 \pm 0.5$ \\
\hline pro & $4.4 \pm 4.4$ & $3.1 \pm 3.8$ & $20.3 \pm 25.6$ & $2.6 \pm 2.9$ & nd \\
\hline gly & $22.3 \pm 8.4$ & $20.6 \pm 7.0$ & $113.9 \pm 40.7$ & $15.7 \pm 3.3$ & $6.4 \pm 1.1$ \\
\hline ala & $9.5 \pm 2.0$ & $8.9 \pm 1.6$ & $63.4 \pm 21.4$ & $8.7 \pm 1.5$ & $2.9 \pm 0.4$ \\
\hline val & $4.1 \pm 2.4$ & $4.1 \pm 3.1$ & $21.2 \pm 9.7$ & $2.8 \pm 0.7$ & nd \\
\hline met & $2.0 \pm 2.2$ & $1.5 \pm 2.0$ & $3.7 \pm 2.6$ & $0.5 \pm 0.2$ & nd \\
\hline ile & $3.1 \pm 2.1$ & $2.8 \pm 1.7$ & $12.8 \pm 4.4$ & $1.8 \pm 0.4$ & $2.6 \pm 0.6$ \\
\hline leu & $2.9 \pm 0.7$ & $2.7 \pm 0.7$ & $22.5 \pm 8.2$ & $3.1 \pm 0.9$ & nd \\
\hline phe & $2.9 \pm 0.9$ & $2.1 \pm 1.4$ & $18.4 \pm 13.2$ & $2.4 \pm 1.1$ & nd \\
\hline tyr & $2.0 \pm 0.7$ & $1.4 \pm 0.9$ & $12.9 \pm 5.4$ & $1.8 \pm 0.6$ & nd \\
\hline his & $8.4 \pm 2.5$ & $7.9 \pm 2.2$ & $62.8 \pm 25.3$ & $9.3 \pm 4.8$ & nd \\
\hline lys & $5.0 \pm 2.4$ & $4.7 \pm 2.1$ & $27.1 \pm 24.6$ & $3.4 \pm 2.2$ & nd \\
\hline $\arg$ & $10.4 \pm 3.2$ & $9.6 \pm 2.2^{a}$ & $51.2 \pm 10.3$ & $7.2 \pm 0.7$ & nd \\
\hline
\end{tabular}

AA amino acid; nd not detectable; ${ }^{a}$ significant difference between $\%($ mole/mole) amount of amino acid sampled by ultrasound and amino acid present in epidermal homogenate $(p<0.05$, student's $t$ test) 

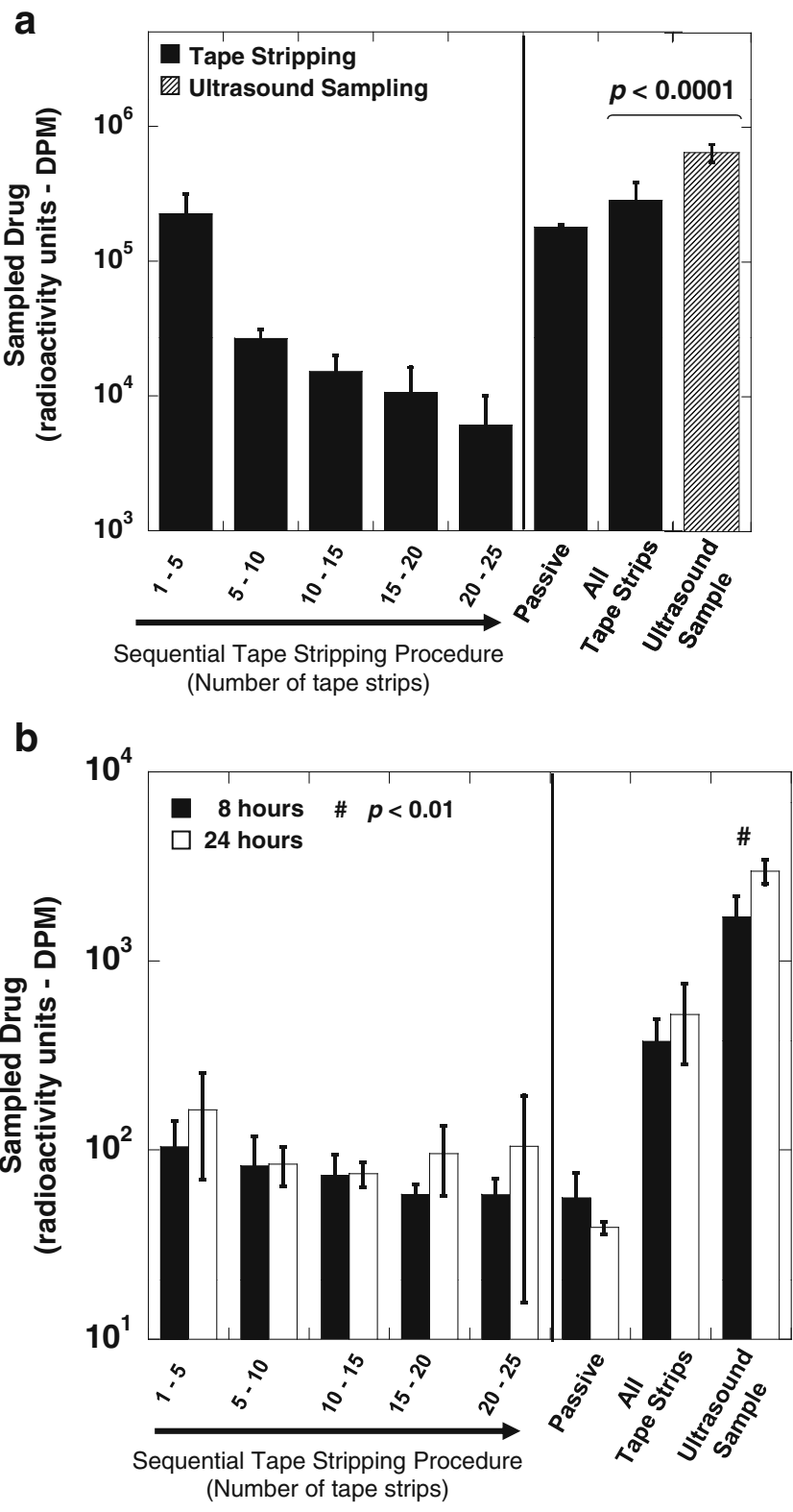

Fig. I In vitro sampling efficiency assessment and comparison of ultrasonic sampling method with tape stripping technique for topical and systemic drug delivery was performed. (a) Docosanol was topically delivered to porcine skin for $4 \mathrm{~h}$. Decreasing amounts of drug were sampled by sequential tape strippings (five strips are grouped together into one data point), implying that most of the drug was accumulated superficially. Cumulative amount of drug sampled by tape stripping technique was found to be significantly less than the drug sampled by ultrasound procedure $(2.42$-fold, $p<0.00 \mathrm{I}) .(n=3)$. (b) To mimic systemic delivery in vitro, docosanol was subdermally delivered from the receiver compartment of diffusion cells for 8 or 24 h. Neither the amount of drug sequentially retrieved by each additional tape stripping, nor the cumulative amount of drug sampled by full tape stripping procedure from skin tissues was statistically different between 8 and $24 \mathrm{~h}$. Ultrasonic sampling was more sensitive than tape stripping in its ability to temporally distinguish between drugs sampled from skin at the two different times. $(n=3)$. that a significant amount of docosanol was delivered into viable epidermis, which was accessible to ultrasound.

To assess the potential of ultrasound to sample systemically delivered drugs, additional experiments were performed in vitro, where systemic delivery was mimicked by including docosanol in the receiver compartment of diffusion cells for either 8 or $24 \mathrm{~h}$ in separate experiments. The skin was sampled by sequential tape stripping and ultrasonic sampling to compare sampling efficiency and temporal sampling resolution of the two methods. Sequential tape stripping yielded relatively constant amounts of docosanol in all tapes, suggesting homogeneous drug distribution in the stratum corneum. Neither the amount of drug sequentially retrieved by each tape, nor the cumulative amount of drug sampled by tape stripping from skin tissues incubated for 8 and $24 \mathrm{~h}$, were statistically different (Student's $t$-test, $n=3$, Fig. 1b). At both time points, however, ultrasonic sampling recovered greater amounts of docosanol compared to tape stripping, and a temporal sampling resolution was attained $(p<0.01$, Student's $t$-test, $n=3$, Fig. 1b). Passive sampling conducted by incubation of sampling buffer with skin retrieved significantly lower amount of docosanol compared with tape stripping and ultrasonic sampling $(p<0.05$, Student's $t$-test, $n=3$, Fig. 1b).

The aim of the in vitro experiments was to demonstrate the proof-of-concept of ultrasonic sampling, and systemic effects such as drug clearance from skin, as relevant in a practical situation, could not be evaluated in the in vitro analysis. Therefore, the effectiveness of ultrasonic sampling was tested in vivo using an anti-fungal drug, fluconazole, which is known to accumulate in skin after intravenous administration $(3,25)$. These experiments were performed in Sprague Dawley rats over a period of seven days. Blood samples were collected to measure plasma fluconazole concentration as a reference. Tape-stripping sampling was performed as a positive control, and skin swabs were used as a passive control. Fluconazole disappeared quickly from blood, and less then $0.15 \%$ of the drug remained in blood after 1 day as compared to its initial amount at $1 \mathrm{~h}$ after intravenous injection. Consistent with preferential partitioning of fluconazole in skin (25), ultrasonic sampling revealed a higher residence time of the drug in skin (Fig. 2). About 30\% of the drug still remained in skin after 7 days compared to its initial amount at $6 \mathrm{~h}$ after drug administration. Recovery of fluconazole from skin by tape stripping was significantly lower than that by ultrasonic sampling over the 7 -day period $(p<0.05$ for sampling at $\geq$ 2 days, Student's $t$ test, $n=9$; Fig. 2). Skin swabs yielded even lower recovery of drug from the skin.

\section{Prolonged Forensic Detection of Cocaine in Skin}

As another potential application, ultrasonic sampling was performed to detect cocaine from skin. Cocaine was intravenously injected in rats, and its concentration in 


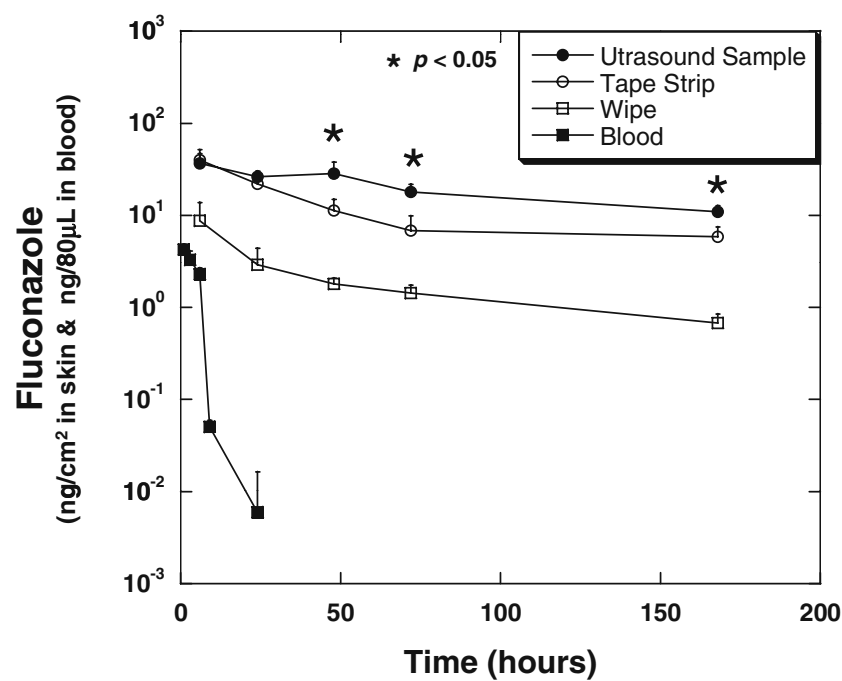

Fig. 2 Quantitative assessment of intravenously administrated fluconazole with ultrasonic sampling method and its comparison with tape stripping technique was performed. Ten $\mathrm{mg} / \mathrm{kg}$ of fluconazole (amounting to $250 \mu \mathrm{Ci} / \mathrm{kg}$ of radioactivity) was intravenously administrated in rats. Fluconazole cocncentration $\left(\mathrm{ng} / \mathrm{cm}^{2}\right)$ in the ultrasonic, tape stripping and swab samples was determined by measuring the total radioactivity $(\mu \mathrm{Ci})$ of the samples and normalizing them by skin sampling area $\left(1.33 \mathrm{~cm}^{2}\right)$. Blood levels of fluconazole (closed squares) rapidly decreased within 24 h; however, ultrasonic sampling (closed circles) revealed prolonged retention of fluconazole in the skin. Fluconazole was detected in the skin by ultrasound for over 7 days, which was also confirmed by tape stripping technique (open circles); however, ultrasound sampled significantly higher amounts of fluconazole than by tape stripping (indicated by $(*): p<0.05$, student's t-test). Skin swabs (open squares) sampled significantly lower amounts of fluconazole than tape stripping and ultrasonic sampling $(p<0.05$, Student's t-test). ( $n=9$, corresponding to three animals and three distinct skin sites within each animal.)

urine was followed for 7 days. Fig. 3 shows the total amount of cocaine present in urine and the entire rat skin (as determined from the amount recovered by ultrasonic sampling from a known area). Less than $0.15 \%$ of cocaine was detected in urine on day 7 compared to the initial amount present on day $1(8 \mathrm{~h})$. Although the amount of drug initially present in urine was 5.87 -fold higher than in skin, a relatively sustained presence of cocaine was seen in skin over a period of 7 days. A high fraction of cocaine $(26.85 \%)$ still remained in skin on day 7 compared to its initial amount at $8 \mathrm{~h}$ after cocaine administration. On day 7 , the amount of cocaine present in urine was significantly lower $(p<0.05$, student's $t$ test, $n=9)$ than the amount measured by passive sampling of skin (wipe). In contrast, ultrasonic sampling revealed about 12-fold higher cocaine presence in skin compared with passive sampling on day 7 .

\section{DISCUSSION}

Ultrasound has been previously used to harvest skin's interstitial fluid in a two-step process-permeabilization followed by vacuum-assisted collection $(21,26,27)$. In this study, we demonstrate that several diagnostically and therapeutically relevant molecules can be rapidly sampled by a brief exposure of ultrasound directly into the ultrasonic coupling medium, thereby avoiding the need to solubilize and disperse analytes in a liquid as is required in conventional methods, including tape stripping.

Table I presents the amount of 16 amino acids sampled by ultrasound and in epidermal homogenate. Except for glutamic acid and arginine, a good correlation between the relative amount of amino acids ( $\%$ mole/mole) in the ultrasonic sample and epidermal homogenate was obtained, which suggests that ultrasonic sampling captured the composition profile of amino acids in the epidermal skin. The concentrations of various amino acids sampled by ultrasound are consistent with the amount sampled by another skin sampling method-reverse iontophoresis (28). Consistent with observations of Bouissou et al. (28), amino acids sampled by ultrasound can be subdivided in two groups: (a) amino acids present at high concentrations in epidermal skin (ser,

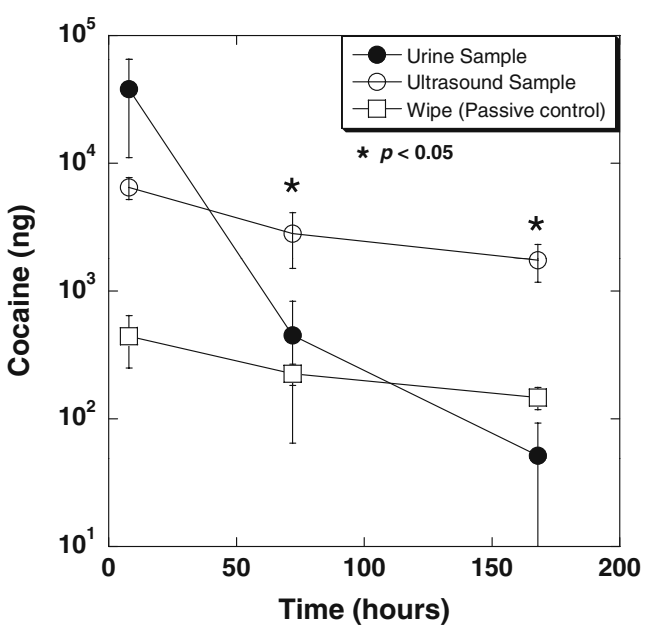

Fig. 3 Forensic detection of cocaine by ultrasonic sampling from skin was assessed. Five $\mathrm{mg} / \mathrm{kg}$ of cocaine (amounting to $175 \mu \mathrm{Ci} / \mathrm{kg}$ of radioactivity) was intravenously administrated in rats. Concentration of cocaine $\left(\mathrm{ng} / \mathrm{cm}^{2}\right)$ in the ultrasonic and swab samples was determined by measuring the total radioactivity $(\mu \mathrm{Ci})$ of the samples and normalizing them by skin sampling area $\left(1.33 \mathrm{~cm}^{2}\right)$. The total amounts of cocaine in skin available to ultrasonic sampling and swabbing were calculated by multiplying respective measured cocaine concentration $\left(\mathrm{ng} / \mathrm{cm}^{2}\right)$ with total body skin area of the rats (determined by mech's formula: $A=k W^{2 / 3}$, where $A$ is the body's skin area in $\mathrm{cm}^{2}, W$ is the body weight in grams and $k=10$ ). The total amount of cocaine in urine was determined by measuring cocaine concentration in urine at specific times and multiplying it by the total urine volume excreted at that time. The total amounts of cocaine present in urine (the conventional sample of choice) and in skin were monitored over a course of 7 days. Cocaine levels fell rapidly to less than $0.15 \%$ of the initial amount in urine (closed circle); however, amount of cocaine in skin that was available to ultrasonic sampling (open circles) showed a sustained presence of the drug in skin for up to 7 days (* indicates $p<$ 0.05 for student's t-test: urine vs. skin drug amount). ( $n=9$, corresponding to three animals and three distinct sites within each animal.) 
gly, arg, ala and his; $22-8$ nmole $/ \mathrm{cm}^{2}$ and (b) amino acids present at moderate to low concentrations (thr, lys, glu, pro, val asp, ile, phe, leu, met and tyr; $\left.5-1 \mathrm{nmole} / \mathrm{cm}^{2}\right)$. Other physiological amino acids were detected neither in ultrasound sample nor in the epidermal homogenate. Seven amino acids (gly, ser, ala, thr, glu, asp and ile) were sampled passively in our study. Except for isoleucine, all passively sampled amino acids were present in a significantly lower concentration than that in the ultrasonic sample ( $p<0.05$, Student's $t$ test, $n=5$, Table I). In contrast, Bouissou et al. reported that all amino acids sampled by reverse iontophoresis were also sampled in comparable amounts passively. This difference can be due to significant difference in passive sampling time between the two studies $(6 \mathrm{~h}$ in the reverse iontophoresis study (28) compared with $5 \mathrm{~min}$ in this study). Nevertheless, the ability to passively sample amino acids suggests intercellular origin of a majority of amino acids; however, further studies are needed to determine the origin of amino acids sampled by ultrasound (intracellular or intercellular).

In addition to amino acids, ultrasound also sampled two other NMFs - lactic acid and urea at concentrations consistent with those previously reported in the literature. Specifically, Nixon et al. (29) reported sampling of about $168 \mathrm{nmoles} / \mathrm{cm}^{2}$ of lactate in $5 \mathrm{~h}$ from porcine skin using reverse iontoporesis, compared with $41.82 \pm 26.65 \mathrm{nmole} / \mathrm{cm}^{2}$ sampled by ultrasound in this study (lactic acid amount was determined from Ref. (29) by integration of iontophoretic molar flux over time). Similarly, ultrasound sampled 13.92 \pm $5.07 \mu \mathrm{mole} / \mathrm{cm}^{2}$ urea, which is in agreement with $5.97 \mu \mathrm{mole} / \mathrm{cm}^{2}$ urea sampled in rat skin by ultrasound/ vacuum-assisted sampling procedure in a separate study (21). Reverse iontophoresis sampled about 40 nmoles $/ \mathrm{cm}^{2}$ in $6 \mathrm{~h}$ from porcine skin (30).

Fig. 1 shows ultrasonic sampling of a model drug, docosanol, delivered to skin either topically or subdermally in vitro. Irrespective of the mode of delivery, ultrasonic sampling collected higher amounts of drug molecules from skin compared to tape stripping. A possible explanation for this observation is that ultrasound collects drugs from the epidermis as opposed to tape stripping, which samples from the stratum corneum. Notably, biophysical effects of ultrasound on skin are known to manifest well into the epidermis (31), suggesting that ultrasound can access molecules present in epidermis. Fig. 1b shows that the difference in the amount of drug present in stratum corneum after $8 \mathrm{~h}$ and $24 \mathrm{~h}$ of subdermal docosanol delivery was statistically insignificant; however, ultrasound sampled significantly more amount of drug from skin at $24 \mathrm{~h}$ compared to $8 \mathrm{~h}$. This difference is likely to originate from the difference in docosanol concentration in the epidermis and dermis layers at $24 \mathrm{~h}$ compared to $8 \mathrm{~h}$, implying that ultrasound samples deeper layers of skin. This result, however, is surprising considering dermis is highly perme- able to hydrophilic solutes. The temporal difference in the amount of docosanol in dermis and epidermis may result from docosanol's high lipophilicity $(\log P=10.5)$ leading to diffusion-limited transport of docosanol in dermis and epidermis. This is supported by 1.66-fold higher amount of docosanal present in skin at $24 \mathrm{~h}$ compared with $8 \mathrm{~h}(n=6)$.

In agreement with in vitro results, Fig. 2 shows that ultrasound offers an attractive means of sampling drugs from deeper layers of skin and over a long period of time. Determination of pharmacokinetic profiles of drugs that therapeutically target deeper viable layers of skin underlying the stratum corneum remains a major focus of US-FDA $(13,32)$; therefore, ultrasonic sampling may provide a patient-friendly and rapid method for determining pharmacokinetic profiles of drugs having therapeutic targets in skin layers other than stratum corneum. However, before reliable quantification of pharmacokinetic profiles can be made, several key parameters of ultrasonic sampling have to be determined. For example, studies that would establish control of ultrasonic sampling depth in the skin, determine ultrasonic sampling efficiency for different layers of skin (stratum corneum, epidermis and dermis), and determine molecule-specific sampling efficiency are warranted. Finally, studies to determine ultrasonic sampling parameters for translation of this study's in vivo results to humans have to be established. One such key parameter is the amount of drug sampled by ultrasound and whether it is above the detection limit of the drug assay. Such extrapolation also requires a consideration for skin/body mass ratio of the animal model and its relationship to that in humans.

Fig. 3 demonstrates that ultrasonic sampling of accumulated illicit drugs in skin can potentially provide significant benefits over current methods, including urinalysis - the present method of choice. Urine sampling has several limitations, including short window of detection (1-3 days) and privacy concerns leading to unsupervised sampling and susceptibility to tampering $(33,34)$. Alternative approaches, such as sweat-collection skin patches and drug detection in hair, have been also proposed (35). Sweat patches provide user convenience but have to be worn for a long time (more than a day), often in unsupervised setting, before detectable quantities of sweat can be collected (34). Additionally, both hair and sweat-patch testing require dispersion of the collected drug in a liquid sample before drug analysis can be performed. This makes these approaches time-intensive and incompatible with onsite testing (36). In contrast, herein, we describe ultrasonic sampling as a rapid method which directly generates a liquefied sample for onsite drug analysis. Ultrasonic sampling can be performed in a supervised setting and bears no privacy concerns during sampling for users, making this method impervious to tampering. As such, skin's high accessibility and sustained presence of drug in skin over a long period of time makes it 
an attractive matrix for forensic detection. However, concerns over skin's susceptibility towards environmental contamination have been presented in a recent study (37) and have to be addressed to make this matrix viable for forensic use. Combinatorial approaches, such as discarding superficial stratum corneum by tape stripping followed by sampling deeper underlying skin tissue by ultrasonic sampling or reverse iontophoresis, may help overcome this challenge.

\section{CONCLUSIONS}

We demonstrate that skin constituents can be directly and rapidly sampled using ultrasound. Ultrasonic exposure to skin at conditions used in this study has a history of clinical safety (38). Studies have shown that the skin barrier recovers to baseline values within $20 \mathrm{~h}$ of ultrasound application (21). Additionally, tolerance of low-frequency ultrasound by patients has also been reported in a number of clinical studies $(39,40)$. Ultrasonic sampling offers several advantages, including non-invasive accessibility to deeper layers of skin and enhanced temporal sampling sensitivity; however, further studies are required to completely establish this method, particularly for conducting pharmacokinetic studies on skin. Future studies must focus on building mechanistic understanding so as to exploit the diverse opportunities that this method provides in the field of diagnostics and therapeutic monitoring.

\section{ACKNOWLEDGMENTS}

We want to acknowledge Anubhav Arora and staff at Animal Resource Center, UCSB for assistance with in vivo experiments.

Open Access This article is distributed under the terms of the Creative Commons Attribution Noncommercial License which permits any noncommercial use, distribution, and reproduction in any medium, provided the original author $(\mathrm{s})$ and source are credited.

\section{REFERENCES}

1. Vickers G. Existence of reservoir in the stratum corneum: experimental proof. Arch Dermatol. 1963;88:20.

2. Joseph Jr R, Oyler J, Wstadik A, Ohuoha C, Cone E. Drug testing with alternative matrices I. Pharmacological effects and disposition of cocaine and codeine in plasma, sebum, and stratum corneum. J Anal Toxicol. 1998;22:6.

3. Wildfeuer A, Faergemann J, Laufen H, Pfaff G, Zimmermann T, Seidl H, et al. Bioavailability of fluconazole in the skin after oral medication. Mycoses. 1994;37:127.

4. Ros A, Wennersten G, Wallin I, Ehrsson H. Concentration of trimethylpsoralen in blood and skin after oral administration. Photo Dermatol. 1988;5:121.
5. Zucchi A, Raho E, Marconi B, Nicoli S, Santini M, Allegra F, et al. Plasma and skin concentration of 5-methoxypsoralen in psoriatic patients after oral administration. J Invest Dermatol. 2001;117: 379-82.

6. Surber C, Smith E, Maibach H. Drug concentration in the skin. In: Bronaugh R, Maibach H, editors. Percutaneous absorption: drugs-cosmetics-mechanisms-methodology. New York: Marcel Dekker; 1999. p. 347.

7. Herkenne C, Alberti I, Naik A, Kalia YN, Mathy FX, Preat V, et al. In vivo methods for the assessment of topical drug bioavailability. Pharm Res. 2008;25:87-103.

8. Elmquist WF, Sawchuk RJ. Application of microdialysis in pharmacokinetic studies. Pharm Res. 1997;14:267-88.

9. Dupuis D, Rougier A, Roguet R, Lotte C, Kalopissis G. In vivo relationship between horny layer reservoir effect and percutaneous absorption in human and rat. J Invest Dermatol. 1984;82:353-6.

10. Bashir SJ, Chew AL, Anigbogu A, Dreher F, Maibach HI. Physical and physiological effects of stratum corneum tape stripping. Skin Res Technol. 2001;7:40-8.

11. Loffler H, Dreher F, Maibach HI. Stratum corneum adhesive tape stripping: influence of anatomical site, application pressure, duration and removal. Br J Dermatol. 2004;151:746-52.

12. Dreher F, Modjtahedi BS, Modjtahedi SP, Maibach HI. Quantification of stratum corneum removal by adhesive tape stripping by total protein assay in 96 -well microplates. Skin Res Technol. 2005;11:97-101.

13. N'Dri-Stempfer B, Navidi WC, Guy RH, Bunge AL. Improved bioequivalence assessment of topical dermatological drug products using dermatopharmacokinetics. Pharm Res. 2009; 26:316-28.

14. Navidi W, Hutchinson A, N'Dri-Stempfer B, Bunge A. Determining bioequivalence of topical dermatological drug products by tape-stripping. J Pharmacokinet Pharmacodyn. 2008; 35:337-48.

15. N'Dri-Stempfer B, Navidi WC, Guy RH, Bunge AL. Optimizing metrics for the assessment of bioequivalence between topical drug products. Pharm Res. 2008;25:1621-30.

16. Kalia Y, Alberti I, Naik A, Guy R. Assessment of topical bioavailability in vivo: the importance of stratum corneum thickness. Skin Pharmacol Appl Skin Physiol. 2001;14:82-6.

17. Herkenne G, Naik A, Kalia YN, Hadgraft J, Guy RH. Dermatopharmacokinetic prediction of topical drug bioavailability in vivo. J Invest Dermatol. 2007;127:887-94.

18. Leboulanger B, Guy R, Delgado-Charro M. Reverse iontophoresis for non-invasive transdermal monitoring. Physiol Meas. 2004;25:35-50.

19. Tezel A, Sens A, Mitragotri S. Investigations of the role of cavitation in low-frequency sonophoresis using acoustic spectroscopy. J Pharm Sci. 2002;91:444-53.

20. Mitragotri S, Blankschtein D, Langer R. Ultrasound-mediated transdermal protein delivery. Science. 1995;269:850.

21. Kost J, Mitragotri S, Gabbay R, Pishko M, Langer R. Transdermal monitoring of glucose and other analytes using ultrasound. Nat Med. 2000;6:347-50.

22. Tezel A, Paliwal S, Shen Z, Mitragotri S. Low-frequency ultrasound as a transcutaneous immunization adjuvant. Vaccine. 2005:23:3800-7.

23. Tabachnick J, LaBadie J. Studies on the biochemistry of epidermis IV. The free amino acids, ammonia, urea, and pyrrolidone carboxylic acid contest of conventional and germ-free albino guinea pig epidermis. J Invest Dermatol. 1970;54:24-31.

24. Rawlings A, Harding C. Moisturization and skin barrier function. Dermatol Ther. 2004;17:43-8.

25. Faegemann J, Godleski J, Laufen H, Liss R. Intracutaneous transport of orally administered fluconazole to the stratum corneum. Acta Derm Venereol. 1995;75:361-3. 
26. Mitragotri S, Coleman M, Kost J, Langer R. Transdermal extraction of analytes using low-frequency ultrasound. Pharm Res. 2000;17:466-70.

27. Mitragotri S, Coleman M, Kost J, Langer R. Analysis of ultrasonically extracted interstitial fluid as a predictor of blood glucose levels. J Appl Physiol. 2000;89:961-6.

28. Bouissou CC, Sylvestre JP, Guy RH, Delgado-Charro MB. Reverse iontophoresis of amino acids: identification and separation of stratum corneum and subdermal sources in vitro. Pharm Res. 2009;26(4):865-71.

29. Nixon S, Sieg A, Delgado-Charro MB, Guy RH. Reverse iontophoresis of L-lactate: in vitro and in vivo studies. J Pharm Sci. 2007;96:3457-65.

30. Wascotte V, Delgado-Charro MB, Rozet E, Wallemacq P, Hubert P, Guy RH, et al. Monitoring of urea and potassium by reverse iontophoresis in vitro. Pharm Res. 2007;24:1131-7.

31. Paliwal S, Menon G, Mitragotri S. Low-frequency sonophoresis: ultrastructural basis for stratum corneum permeability assessed using quantum dots. J Invest Dermatol. 2006;126: 1095-101

32. U. FDA. Guidance for industry on special protocol assessment; availability. Fed Regist. 67:35122 (2002).
33. Hawks R, Chiang C. Urine testing for drugs of abuse. No: DHHS (ADM) 87-1481:129 (1986).

34. Dolan K, Rouen D, Kimber J. An overview of the use of urine, hair, sweat and saliva to detect drug use. Drug Alcohol Rev. 2004; 23:213-7.

35. Cone E. Legal, workplace, and treatment drug testing with alternate biological matrices on a global scale. Forensic Sci Int. 2001;121:7-15.

36. Rivier L. Techniques for analytical testing of unconventional samples. Best Pract Res Clin Endocrinol Metab. 2000;14:147-65.

37. Kidwell D, Kidwell J, Shinohara F, Harper C, Roarty K, Bernadt $\mathrm{K}$, et al. Comparison of daily urine, sweat, and skin swabs among cocaine users. Forensic Sci Int. 2003;133:63-78.

38. Ogura M, Paliwal S, Mitragotri S. Low-frequency sonophoresis: current status and future prospects. Adv Drug Deliv Rev. 2008;60:1218-23.

39. Katz NP, Shapiro DE, Herrmann TE, Kost J, Custer LM. Rapid onset of cutaneous anesthesia with EMLA cream after pretreatment with a new ultrasound-emitting device. Anesth Analg. 2004;98:371-6. table of contents.

40. Becker BM, Helfrich S, Baker E, Lovgren K, Minugh PA, Machan JT. Ultrasound with topical anesthetic rapidly decreases pain of intravenous cannulation. Acad Emerg Med. 2005;12:289-95. 